\title{
Lesiones producidas en una pista temporal de patinaje sobre hielo
}

\author{
Víctor Jesús Moreno Alcaraz y Pedro Ángel López Miñarro
}

Departamento de Educación Física. Universidad de Murcia. Murcia, España.

\section{RESUMEN}

Introducción y objetivos: La práctica del patinaje sobre hielo conlleva un aumento del riesgo de lesiones debido a una mayor probabilidad de caídas. El objetivo de este estudio fue describir las lesiones producidas y sus factores de riesgo en una pista temporal de patinaje sobre hielo.

Métodos: Se registraron las lesiones producidas en una pista de hielo instalada durante un mes en un municipio de Murcia. Para cada uno de los patinadores lesionados, se registraron datos sobre edad, sexo, localización de la lesión, mecanismo lesional, diagnóstico de la lesión y experiencia como patinador. Los datos fueron registrados por el personal de la Cruz Roja Española en el puesto de primeros auxilios ubicado junto a la pista.

Resultados: Un total de 185 sujetos $(95$ mujeres y 90 varones) presentaron algún tipo de lesión. Las lesiones más comunes fueron de carácter leve, como contusiones (40\%) y heridas superficiales $(28,2 \%)$. Las fracturas representaron el I,6\% de las lesiones. La mayoría de las lesiones se localizaron en la muñeca $(29,2 \%)$, seguida del pie $(13,0 \%)$ y la rodilla $(10,8 \%)$. El principal mecanismo de lesión fue la caída (71\%), debida principalmente a la pérdida de equilibrio $(61,2 \%)$. Los sujetos $\sin$ experiencia en patinaje sobre hielo presentaron mayor frecuencia de lesiones que los patinadores más experimentados.

Conclusiones: El número de lesiones ocurridas en la pista de hielo fue bajo en relación con el número total de patinadores que la visitaron, y en su mayoría éstas fueron de carácter leve.

PALABRAS CLAVE: Lesión. Patinaje sobre hielo. Pista de hielo. Prevención. Patinador recreativo.

\begin{abstract}
Introduction and objectives: Recreational ice-skating carries a high risk of injury because of the strong probability of falls. The aim of this study was to describe injuries and associated risk factors in a temporary ice rink.

Methods: We recorded all injuries produced in a temporary ice rink installed for I month in a town in Murcia. For all ice skaters injured, information on age, gender, body region injured, mechanism of injury, diagnosis, and ice skating experience was recorded. The data were gathered by staff from the Spanish Red Cross in the first aid facilities situated beside the ice rink.

Results: A total of 185 recreational ice skaters (95 females and 90 males) were injured. The most common injuries were slight injuries such as bruises (40\%) and superficial wounds (28.2\%). Fractures accounted for $1.6 \%$ of all injuries. Most skating-related injuries involved the wrist (29.2\%), followed by the foot (I3.0\%) and the knee (I0.8\%). Most of the injuries were caused by falls (71\%), mainly due to loss of balance (61.2\%). Beginners appeared to be more prone to injury than experienced skaters.

Conclusions: The number of injuries was low compared with the total number of skaters and most of the injuries were slight.
\end{abstract}

KEY WORDS: Injury. Ice skating. Ice rink. Safety. Recreational skater. 


\section{INTRODUCCIÓN}

En los últimos años es habitual la instalación de pistas de patinaje sobre hielo con carácter temporal, sobre todo durante el período de Navidad. La presencia de una pista de hielo supone una gran novedad para la población de la zona y ofrece la oportunidad de practicar una actividad atractiva y poco habitual. Sin embargo, la práctica del patinaje sobre hielo supone un alto riesgo de padecer lesiones, sobre todo fracturas, para los patinadores "ocasionales" con poca o ninguna experiencia en el patinaje sobre hielo ${ }^{1-6}$.

Diversos estudios internacionales han analizado las lesiones producidas en pistas de hielo, mostrando grandes diferencias en cuanto al número de sujetos lesionados, tipo de lesiones y su localización. No obstante, la mayoría de estos estudios coinciden en señalar que las lesiones más frecuentes en las pistas de hielo son de carácter grave, sobre todo fracturas localizadas en los miembros superiores, señalando la caída como principal mecanismo de lesión. Además, estos estudios encuentran que la mayoría de los sujetos lesionados son jóvenes de entre 10 y 14 años de edad, y no se encuentran grandes diferencias entre uno y otro sexo ${ }^{5,7-11}$. Algunos autores consideran el patinaje recreativo sobre hielo como una actividad de gran potencial lesivo ${ }^{6,8}$.

No conocemos estudios que hayan analizado la frecuencia de lesiones y sus mecanismos de producción en población española. El objetivo de este estudio fue describir las lesiones producidas en una pista temporal de hielo, así como sus mecanismos de producción.

\section{MÉTOdos}

Un total de 9.000 patinadores recreativos visitaron la pista de patinaje sobre hielo instalada en un municipio de Murcia, desde el 15 de diciembre de 2006 hasta el 15 de enero de 2007 , coincidiendo con las vacaciones escolares de Navidad.

Todos los sujetos que padecieron alguna lesión en la pista de hielo como consecuencia de la práctica del patinaje fueron atendidos por el personal de la Cruz Roja Española en el puesto de primeros auxilios ubicado junto a la propia pista. De cada persona lesionada se realizó un parte de accidente donde se registraron los datos personales del accidentado tales como nombre, edad y sexo, junto con la localización de la lesión y un diagnóstico previo. Adicionalmente, a todos ellos se les solicitó que realizaran un cuestionario que proporcionaba información sobre la frecuencia de práctica de esta actividad y el mecanismo de producción de la lesión. Sesenta y nueve de ellos accedieron a realizar el cuestionario.
A nivel estadístico se realizó un análisis descriptivo en base a frecuencias, así como un test de $\chi^{2}$ para comparar la frecuencia de lesiones entre sexos y en base a la experiencia previa de los patinadores. Los datos fueron analizados con el programa estadístico SPSS (versión 14.0) y se estableció un valor de $\mathrm{p}<$ 0,05 para la significación estadística.

\section{Resultados}

Un total de 185 sujetos (95 mujeres y 90 varones) con edades comprendidas entre los 3 y los 45 años de edad padecieron algún tipo de lesión, y el rango de edad comprendido entre los 10 y 12 años fue el que evidenció mayor frecuencia de lesiones (fig. 1). No encontramos diferencias significativas en la distribución de proporciones en base al sexo $\left(\chi^{2}=0,153\right.$; $\left.\mathrm{p}=0.71\right)$.

Las lesiones más comunes fueron de carácter leve, tales como contusiones y heridas superficiales, seguidas de esguinces o distensiones y cortes. Se produjeron 3 fracturas, que significaron el 1,6\% de las lesiones totales (tabla I). De todos los lesionados, 15 tuvieron que ser trasladados al centro de salud local o al hospital Virgen de la Arrixaca de Murcia, para establecer un mejor diagnóstico y un tratamiento adecuado.

De las 185 lesiones registradas, 83 se localizaron en la extremidad superior y 71 en la extremidad inferior. La parte del cuerpo más lesionada fue la muñeca, seguida del pie y de la rodilla (fig. 2).

La mayoría de las rozaduras y heridas superficiales localizadas en los pies, en los tobillos y en las piernas se produjeron por

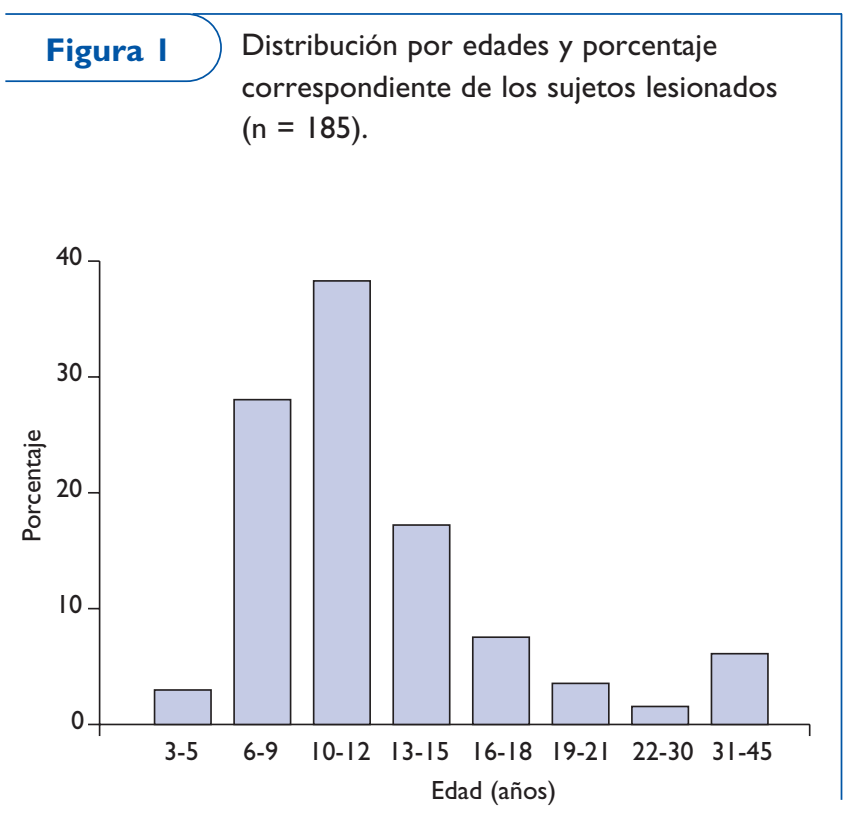


TRABAJOS ORIGINALES

Tabla I Número y porcentaje de las lesiones producidas en la pista de hielo $(n=185)$

\begin{tabular}{|lcc|}
\hline Diagnóstico de la lesión & $\mathbf{n}$ & Porcentaje \\
\hline Contusión & 74 & 40,0 \\
\hline Corte & 10 & 5,5 \\
\hline Esguince/distensión & 37 & 20,0 \\
\hline Fractura & 3 & 1,6 \\
\hline Heridas superficiales & 52 & 28,2 \\
\hline Traumatismo craneoencefálico & 3 & 1,6 \\
\hline Contractura & $\mathrm{I}$ & 0,5 \\
\hline Luxación & $\mathrm{I}$ & 0,5 \\
\hline Mareo & 2 & 1,1 \\
\hline Escocedura ojos & $\mathrm{I}$ & 0,5 \\
\hline Crisis nerviosa & $\mathrm{I}$ & 0,5 \\
\hline Total & 185 & 100,0 \\
\hline
\end{tabular}

Figura 2

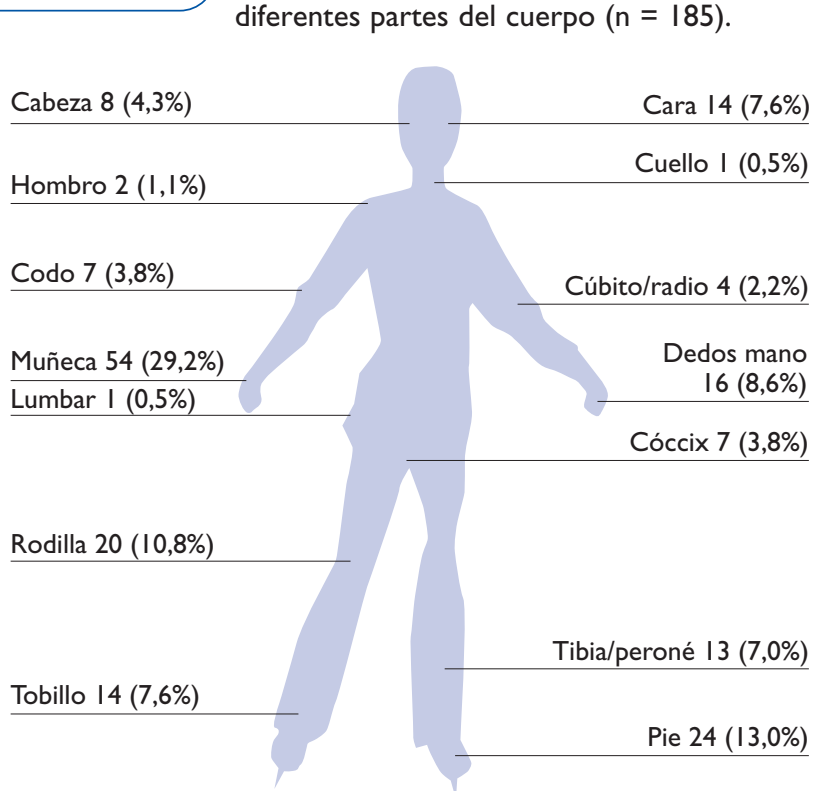

llevar calcetines cortos y por un mal ajuste de los patines. Las heridas y los cortes en las manos fueron ocasionados en su mayoría al ser pisados por la cuchilla de otro patinador. El principal mecanismo de lesión fue la caída, debida principalmente a la pérdida de equilibrio (tablas II y III).

La mayoría de los patinadores lesionados tenían muy poca o ninguna experiencia en patinaje sobre hielo. Solamente 5 personas habían patinado más de 5 veces antes de producirse la Tabla II Causas de caída de los sujetos lesionados
$(\mathrm{n}=49)$

\begin{tabular}{|lcc|}
\hline Causas de la caída & $\mathbf{n}$ & Porcentaje \\
\hline Pérdida de equilibrio & 30 & 61,2 \\
\hline Empujado por otro patinador & $1 \mathrm{I}$ & 22,5 \\
\hline Tropiezo & 7 & 14,3 \\
\hline No esquivar a tiempo & $\mathrm{I}$ & 2,0 \\
\hline Total & 49 & 100,0 \\
\hline
\end{tabular}

lesión, y los que patinaban por primera vez fueron los que presentaron una significativa $(\mathrm{p}<0.001)$ mayor frecuencia de lesiones (fig. 3).

\section{Discusión}

La práctica de una actividad como el patinaje sobre hielo en una pista temporal supone un aumento del riesgo de accidente por diversos motivos, tales como la inexperiencia de los usuarios (niños y adolescentes, principalmente), los problemas de estabilidad por la reducida base de sustentación, el reducido coeficiente de rozamiento de la pista, etc. A pesar de ello, en nuestro estudio sólo se lesionaron el 2,05\% del total de usuarios que hicieron uso de la instalación durante el mes que estuvo en funcionamiento. Existe una gran variabilidad en el número de personas lesionadas en estudios previos realizados en patinadores recreativos sobre hielo. Así Johannsen et $\mathrm{al}^{7}$ refieren un total de 105 sujetos lesionados durante un año, mientras que Lam et $\mathrm{al}^{12}$ encontraron tan sólo 43 lesionados en un período de 2 años y 3 meses. Clarke et al $^{5}$ registraron un total de 125 sujetos lesionados en un período de 6 semanas, y las lesiones más frecuentes fueron las fracturas y luxaciones en los miembros superiores. Por el contrario, McGeehan et $\mathrm{al}^{10}$ encontraron una mayor localización de lesiones en los miembros inferiores, en la cabeza y en la cara. A partir de estos resultados es difícil establecer un patrón estándar sobre los tipos de lesiones y su localización, así como considerar el patinaje sobre hielo como una actividad con un gran riesgo de generar lesiones. No obstante, una limitación de nuestro estudio radica en que, probablemente, algunos usuarios de la pista padecieron accidentes de poca consideración que provocaron pequeñas lesiones a las que no dieron la importancia oportuna y decidieron no hacer uso del servicio de primeros auxilios.

En coincidencia con la mayoría de estudios, el rango de edad entre los 10 y 12 años es el que evidencia una mayor frecuencia 
TRABAJOS ORIGINALES

Tabla III Distribución y porcentaje de lesiones según el mecanismo que las produjo $(\mathrm{n}=69)$

\begin{tabular}{|lccccccc|}
\hline Mecanismo lesional & $\begin{array}{c}\text { Contusión } \\
\mathbf{n}(\%)\end{array}$ & $\begin{array}{c}\text { Cortes } \\
\mathbf{n}(\%)\end{array}$ & $\begin{array}{c}\text { Contracturas } \\
\mathbf{n}(\%)\end{array}$ & $\begin{array}{c}\text { Esguincel } \\
\text { distensión } \\
\mathbf{n}(\%)\end{array}$ & $\begin{array}{c}\text { Fracturas } \\
\mathbf{n}(\%)\end{array}$ & $\begin{array}{c}\text { Heridas } \\
\text { superficiales } \\
\mathbf{n}(\%)\end{array}$ & $\begin{array}{c}\text { Total } \\
\mathbf{n}(\%)\end{array}$ \\
\hline Caída hacia atrás & $16(50,0)$ & $3(50,0)$ & $0(0,0)$ & $6(66,7)$ & $0(0,0)$ & $2(10,5)$ & $28(40,5)$ \\
\hline Caída hacia delante & $11(34,4)$ & $I(16,0)$ & $0(0,0)$ & $3(33,4)$ & $1(100)$ & $5(26,3)$ & $21(30,5)$ \\
\hline Choque contra otro patinador & $3(9,4)$ & $0(0,0)$ & $0(0,0)$ & $0(0,0)$ & $0(0,0)$ & $1(5,3)$ & $4(5,8)$ \\
\hline Choque contra la valla & $1(3,1)$ & $0(0,0)$ & $1(100)$ & $0(0,0)$ & $0(0,0)$ & $1(5,3)$ & $3(4,4)$ \\
\hline $\begin{array}{l}\text { Mal ajuste patines/calcetines } \\
\text { inadecuados }\end{array}$ & $0(0,0)$ & $0(0,0)$ & $0(0,0)$ & $0(0,0)$ & $0(0,0)$ & $10(52,6)$ & $10(14,4)$ \\
\hline Pisado por un patín & $1(3,1)$ & $2(34,0)$ & $0(0,0)$ & $0(0,0)$ & $0(0,0)$ & $0(0,0)$ & $3(4,4)$ \\
\hline Total & $32(100)$ & $6(100)$ & $1(100)$ & $9(100)$ & $1(100)$ & $19(100)$ & $69(100)$ \\
\hline
\end{tabular}

de lesiones, sin diferencias en base al sexo ${ }^{5,7-11}$. La caída fue el principal mecanismo de lesión, y la muñeca fue la parte del cuerpo lesionada con mayor frecuencia. Este hecho se debe a que las caídas en patinaje sobre hielo suelen ser hacia delante, por lo que los patinadores colocan sus manos delante del cuerpo para frenar la caída. Además, los sujetos con menor experiencia en patinaje sobre hielo fueron los que más se lesionaron ${ }^{5,7-12}$.

La mayoría de estudios previos realizados acerca de las lesiones en patinadores recreativos sobre hielo encuentran una alta frecuencia de lesiones de carácter grave, sobre todo fracturas ${ }^{1,25,6,8,9,12}$, y consideran la práctica del patinaje sobre hielo como una actividad de alto potencial lesivo ${ }^{6,8}$. En esta línea, estudios comparativos entre patinadores sobre hielo y patinadores sobre ruedas concluyen que el patinaje sobre hielo presenta un mayor riesgo de causar lesiones graves, sobre todo fracturas, que el patinaje sobre ruedas,10,11. En nuestro estudio, en coincidencia con Williamson y Lowdon ${ }^{2}$ y Johannsen et $\mathrm{al}^{7}$, encontramos que la mayoría de lesiones producidas en patinaje recreativo sobre hielo son de carácter leve, tales como contusiones y heridas superficiales, aunque también se producen lesiones más graves, como las fracturas óseas, si bien su frecuencia es muy reducida $(1,6 \%)$. Un factor que podría explicar las diferencias entre este estudio y otros que encuentran una alta frecuencia de fracturas es la velocidad media del patinador, si bien esta variable no ha sido registrada en estudio alguno.

Por otro lado, cabe destacar la alta proporción de lesiones localizadas en la cabeza y en la cara encontrada en nuestro estudio. Estos resultados se pueden atribuir a las características del hielo, donde las caídas hacia delante producen más lesiones

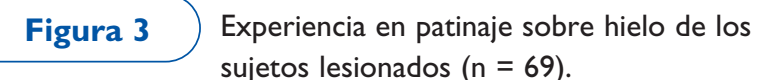
sujetos lesionados $(n=69)$.

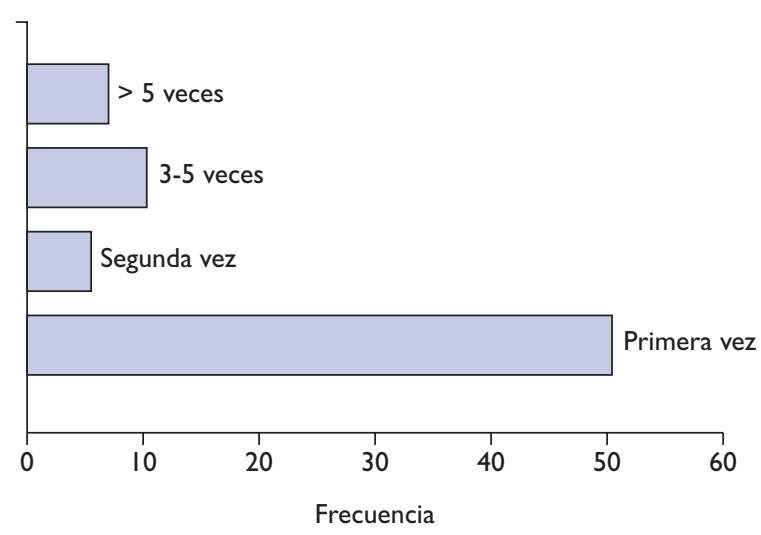

en la cara, boca, dientes y cabeza que en el patinaje sobre ruedas, ya que el intento de frenar la caída con las manos no es suficiente, por lo que en esta acción se involucran la cabeza y la cara, que golpea contra la superficie helada ${ }^{10,13}$.

Tomando como referencia los datos de este trabajo, proponemos una serie de pautas para minimizar o evitar los mecanismos de producción de las lesiones en las pistas de patinaje sobre hielo:

- Uso de protecciones tales como muñequeras con superficie no deslizante, rodilleras, coderas y casco, a ser posible con rejilla para proteger la cara.

- Obligatoriedad de llevar guantes para evitar cortes en los dedos. 
- Limitar el número de patinadores en pista, para evitar choques y tropiezos.

- Establecer una zona delimitada para principiantes y separada del resto de usuarios, donde puedan aprender a pati-nar sin riesgo de ser atropellados por patinadores más rápidos.

- Presencia de monitores cualificados que enseñen las técnicas básicas de patinaje, tales como posición básica, equilibrio, primeros pasos, frenada, giros, caída y puesta en pie, así como algunas medidas de actuación para evitar accidentes mientras patinan, como respetar las normas de la pista, patinar por zonas de poco tránsito, levantarse rápido del suelo en caso de caída, colocar las manos cerca del cuerpo en caso de caída.
- Establecer normas de comportamiento en la pista, como patinar todos en el mismo sentido, no patinar a velocidades altas, no hacer cadenas de patinadores, no empujar, etc.

\section{CONCLUSIONES}

El número de lesiones producidas en una pista temporal de patinaje sobre hielo, instalada durante un mes, fue bajo en relación con el número total de patinadores que la visitaron, y en su mayoría fueron de carácter leve. Los sujetos sin experiencia en patinaje sobre hielo presentan mayor frecuencia de lesiones que los patinadores experimentados.
1. Horner C, McCabe MJ. Ice skating and roller disco injuries in Dublin. Br J Sports Med. 1984;18:207-11.

2. Williamson DM, Lowdon IM. Ice skating injuries. Injury. 1986;17:205-7.

3. Radford PJ, Williamson DM, Lowdon IM. The risks or injury in public ice skating. Br J Sports Med. 1986;22:78-80.

4. Oakland CD. Ice skating injuries: can they be reduced or prevented? Arch Emerg Med. 1990;7:95-9.

5. Clarke HJ, Ryan D, Cullen I, Cusack S. The impact of a temporary ice-rink on an emergency department service. Eur J Emerg Med. 2006;13:204-8.

6. Dillon JP, Geurin S, Laing AJ, Ryan D, Dolan M. The impact of ice skating injuries on orthopaedic admissions in a regional hospital. Ir Med J. 2006;99:7-8.

7. Johannsen HG, Mikkelsen JB, Fricke A. Skating injuries. A study of an injury sample in a Danish community skating rink. Nord Med. 1997;112:61-2.
8. Bernard AA, Corlett S, Thomsen E, Bell N, McMahon A, Richmond $\mathrm{P}$, et al. Ice skating accidents and injuries. Injury. 1988;19:191-2.

9. Murphy NM, Riley P, Keys C. Ice skating injuries to the hand. J Hand Surg [Br]. 1990;15:349-51.

10. McGeehan J, Shields BJ, Smith GA. Children should wear helmets while ice skating: a comparison of skating related injuries. Pediatrics. 2004;114:124-8.

11. Knox CL, Comstock RD, McGeehan J, Smith, GA. Differences in the risk associated with head injury for pediatric ice skaters, roller skaters, and inline skaters. Pediatrics. 2006;118:549-54.

12. Lam CK, Leung WY, Wu WC, Lam J, Ip FK. Orthopaedic ice skating injuries in a regional hospital in Hong Kong. Hong Kong Med J. 1997;3:131-4.

13. Knox CL, Comstock RD. Video analysis of falls experienced by paediatric ice skaters and roller/in line skaters. Br J Sports Med. 2006;40:268-71. 